\title{
INFLUÊNCIA DA CONCENTRAÇÃO MOLAR DA SOLUÇÃO ATIVADORA E DA RAZÃO ATIVADOR/PRECURSOR NA RESISTÊNCIA À COMPRESSÃO DE ARGAMASSAS GEOPOLIMÉRICAS*
}

\author{
Greisson Peixoto Rangel ${ }^{1}$ \\ Dylmar Penteado Diasr ${ }^{2}$ \\ Alexandre Magno Alves de Oliveira ${ }^{3}$ \\ Afonso Rangel Garcez Azevedo ${ }^{4}$ \\ Sergio Neves Monteiro ${ }^{5}$ \\ Markssuel Teixeira Marvilar ${ }^{6}$
}

\section{Resumo}

A variação da resistência à compressão de argamassas geopoliméricas foi estudada em função da razão ativador/precursor e da molaridade da solução ativadora. As argamassas estudadas foram confeccionadas com metacaulim (precursor) ativado com solução composta por hidróxido de potássio e água destilada, e areia normal brasileira. De acordo com os resultados, a resistência aumenta com a diminuição da razão ativador/precursor e com o aumento da molaridade da solução ativadora. Além disso, os valores encontrados condizem com os presentes da literatura e com possíveis aplicações para argamassas de múltiplo uso.

Palavras-chave: Argamassa; Geopolímero; Resistência; Ativação alcalina.

\section{INFLUENCE OF MOLAR ACTIVATION SOLUTION CONCENTRATION AND ACTIVATOR/PRECURSOR RATIO ON COMPRESSIVE STRENGTH OF GEOPOLYMERIC MORTARS.}

\begin{abstract} literature review and the possible uses of multiple use mortar.

Keywords: Mortar; Geopolymer; Strength; Alkaline activation.
\end{abstract}

The compressive strength variation of geopolymeric mortars was studied according to the activator/precursor ratio and the molarity of the activator solution. The mortars were made with metacaulim (precursor) activated by potassium hydroxide and destilled water solution, and normal brazilian sand. According to the results, the strength grows with the diminish of activator/precursor ratio and with the increase of activation solution molarity. Besides that, the results are in consonance with the

1 Engenheiro Civil, mestrando em Estruturas, LECIV, Universidade Estadual do Norte Fluminense Darcy Ribeiro, Campos dos Goytacazes, RJ, Brasil.

2 Engenheiro Civil, Doutor, Professor Associado, LECIV, Universidade Estadual do Norte Fluminense Darcy Ribeiro, Campos dos Goytacazes, RJ, Brasil.

3 Engenheiro Civil, mestrando em Estruturas, LECIV, Universidade Estadual do Norte Fluminense Darcy Ribeiro, Campos dos Goytacazes, RJ, Brasil.

4 Engenheiro Civil, doutorando em Estruturas, LECIV, Universidade Estadual do Norte Fluminense Darcy Ribeiro, Campos dos Goytacazes, RJ, Brasil.

5 Engenheiro Metalúrgico, Doutor, Professor Titular, Laboratório de Ciência dos materiais, Instituto Militar de Engenharia, Rio de Janeiro, RJ, Brasil.

6 Engenheiro Civil, mestrando em Estruturas, LECIV, Universidade Estadual do Norte Fluminense Darcy Ribeiro, Campos dos Goytacazes, RJ, Brasil. 


\section{INTRODUÇÃO}

A busca por sustentabilidade na construção civil, seja por razões ecológicas ou econômicas, contempla o uso de materiais menos agressivos ao meio ambiente e com melhor desempenho. Seja pelo impacto do processo de fabricação ou pela capacidade de prover estruturas cada vez mais duráveis, o desenvolvimento de novos materiais possui papel cada vez mais decisivo na Construção Civil.

No Brasil, apenas em 2016, foram produzidas, segundo estimativas do Sindicato Nacional da Indústria do Cimento, cerca de 43.702.432 toneladas de cimento Portland [1], valor que motiva os estudos da substituição parcial ou completa do cimento Portland, seja por resíduos ou materiais alternativos.

Nesse contexto, este trabalho apresenta o estudo inicial da influência da relação entre ativador e precursor na resistência à compressão de argamassas geopoliméricas ativadas com $\mathrm{KOH}$.

O termo geopolímero foi criado na década de 1970 pelo Cientista e Engenheiro francês Joseph Davidovits, e aplicado a uma classe de materiais sólidos sintetizados pela reação de materiais aluminossilicatos com uma solução alcalina [2].

De acordo com De Weerdt [3], um geopolímero é um aglomerante inorgânico. É sintetizado pela mistura, em temperatura ambiente ou maior, de um pó aluminossilicato reativo (cinza volante, metacaulim, argila calcinada, entre outros) chamado de precursor, com um ativador alcalino que contenha hidróxidos, silicatos, aluminatos, carbonatos, sulfatos alcalinos ou uma combinação destes. O produto dessa reação é, principalmente, uma fase amorfa ou praticamente amorfa de aluminossilicato constituído de $\mathrm{SiO}_{4}{ }^{4-}$ e $\mathrm{AlO}_{4}{ }^{5-}$ tetraédricos interligados, formando uma estrutura polimérica tridimensional (Figura 1).
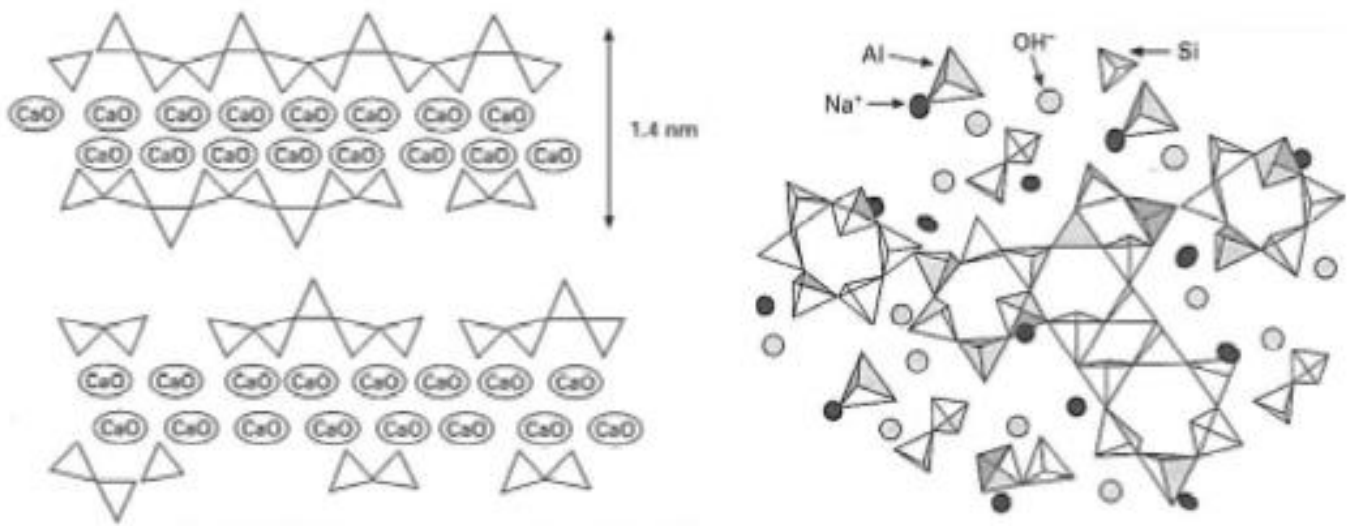

Figura 1. Comparação entre o produto da reação de hidratação o cimento Portland C-S-H (esquerda) e o produto da reação de formação de um geopolímero (direita) [3].

Dos possíveis precursores, este trabalho utilizou o metacaulim, o qual é obtido por meio da calcinação de argila caulinítica em temperaturas entre $400^{\circ} \mathrm{C}$ e $950^{\circ} \mathrm{C}$. Nesse processo, a caulinita tem seu arranjo cristalino desfeito. O produto formado é denominado metacaulinita, apresentando na difração de raios $x$ uma estrutura amorfa [4]. Metaucaulim é, portanto, o produto comercial composto predominantemente por metacaulinita.

Quanto à solução ativadora, a composição mais comum na literatura é a combinação de um silicato alcalino com um hidróxido alcalino, principalmente o silicato e o hidróxido de sódio. A ativação por meio apenas de hidróxidos, seja de sódio ou potássio, apesar de resultar em valores mais baixos de resistência à 
compressão, pode ser útil para a produção de uma argamassa geopolimérica [5] para variados usos.

\section{MATERIAIS E MÉTODOS}

Os materiais utilizados foram o metacaulim (precursor), $\mathrm{KOH}$ (ativador), água destilada e agregado miúdo.

O metacaulim utilizado foi fornecido pela Metacaulim do Brasil Ltda. Sua composição química, obtida por meio de EDX, pode ser observada na Tabela 1.

Tabela 1. Composição química em termos de óxidos.

\begin{tabular}{|c|c|}
\hline Composto & Quantidade (\%) \\
\hline $\mathrm{SiO}_{2}$ & 53,254 \\
\hline $\mathrm{Al}_{2} \mathrm{O}_{3}$ & 38,939 \\
\hline $\mathrm{K}_{2} \mathrm{O}$ & 3,389 \\
\hline $\mathrm{Fe}_{2} \mathrm{O}_{3}$ & 1,599 \\
\hline $\mathrm{TiO}_{2}$ & 1,354 \\
\hline $\mathrm{SO}_{3}$ & 1.348 \\
\hline Outros & 0,117 \\
\hline
\end{tabular}

Como a única fonte de óxidos de silício e alumínio é o metacaulim, com sua composição química é possível calcular a razão $\mathrm{SiO}_{2} / \mathrm{Al}_{2} \mathrm{O}_{3}$, em massa, da argamassa geopolimérica, que foi igual a 1,37.

Para o preparo da solução ativadora foi utilizado o hidróxido de potássio P.A (para análise) e água destilada.

O agregado miúdo escolhido foi a areia normal brasileira fornecida pelo IPT (Instituto de Pesquisas Tecnológicas). Em cada traço, a quantidade de agregado foi dividida igualmente entre as quatro frações granulométricas nas quais a areia normal brasileira é fornecida: grossa (\#16), média grossa (\#30), média fina (\#50) e fina (\#100).

Para estudar os efeitos da variação da composição das argamassas geopoliméricas na resistência à compressão, três corpos de prova de cada traço foram moldados, desmoldados aos três dias e rompidos por compressão aos sete dias, com utilização de uma prensa manual da fabricante Solotest com capacidade máxima de $100 \mathrm{tf}$.

Os dois principais parâmetros da composição que afetam a resistência à compressão são a razão ativador/precursor, relação das massas do $\mathrm{KOH}$ e do metacaulim utilizados, e a concentração molar da solução ativadora, a qual também se relaciona diretamente com a razão água/sólidos.

Dessa forma, três composições iniciais foram estudadas, assim como valores da literatura, para determinar valores razoáveis da razão ativador/precursor, a saber: 1:1:3:0,57; 1:075:3:0,48; 1:0,5:3:0,37 (metacaulim: $\mathrm{KOH}$ : areia: água, em massa).

Posteriormente, a razão ativador/precursor com melhor resultado foi mantida constante enquanto a quantidade de água foi alterada, de forma a proporcionar diferentes molaridades para a solução ativadora. A nomenclatura utilizada para as diferentes composições foram: $\mathrm{K}$ seguida pela razão ativador/precursor e da razão água/sólidos, separadas por uma barra, ou seja, K1/0,57; K0,75/0,48 e K0,5/0,37. 


\section{RESULTADOS E DISCUSSÃO}

A resistência à compressão em função da razão ativador/precursor pode ser vista na Tabela 2 e na Figura 2:

Tabela 2. Resistência à compressão das composições iniciais.

\begin{tabular}{|c|c|}
\hline Nomenclatura & $\begin{array}{c}\text { Resistência à } \\
\text { compressão (MPa) }\end{array}$ \\
\hline $\mathrm{K} 1 / 0,57$ & 0,4 \\
\hline $\mathrm{K} 0,75 / 0,48$ & 1,2 \\
\hline $\mathrm{K} 0,5 / 0,37$ & 4,3 \\
\hline
\end{tabular}

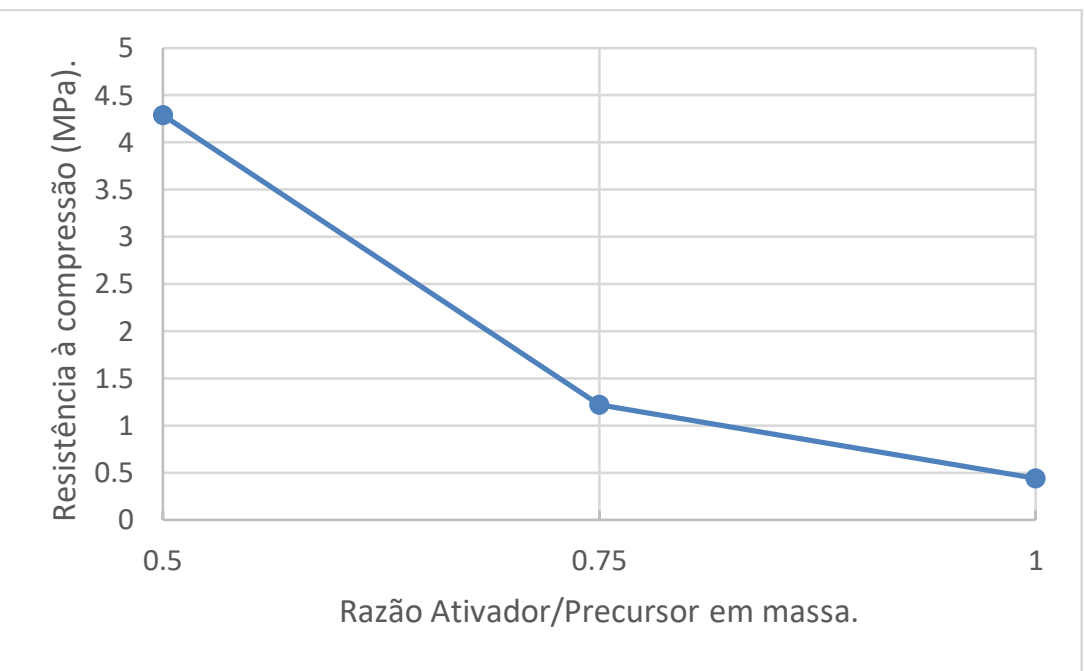

Figura 2. Resistência à compressão em função da razão ativador/precursor.

Como pode ser observado na Tabela 2 e na Figura 2, o traço K0,5/0,37 é o que alcançou maior resistência mecânica. No entanto, não é possível afirmar categoricamente que a resistência à compressão aumenta com a diminuição da razão ativador/precursor, pois a diferença nas molaridades dos traços pode ter efeito relevante quanto à resistência final das argamassas.

Assim, a razão ativador/precursor da argamassa com maior resistência à compressão, K0,5/0,37, foi mantida e a molaridade da solução foi alterada por meio da variação da quantidade de água e, portanto, da razão água/sólidos. Foram, então, feitas as seguintes composições: K0,5/0,48; K0,5/0,5; K0,5/0,52; K0,5/0,54 e K0,5/0,56. Os resultados obtidos podem ser observados na Tabela 3 e na Figura 3.

Tabela 3. Resistência à compressão dos traços com razão ativador/precursor igual a 0,5.

\begin{tabular}{|c|c|c|}
\hline $\begin{array}{c}\text { Razão } \\
\text { água/sólidos }\end{array}$ & $\begin{array}{c}\text { Molaridade } \\
\text { (mols/l) }\end{array}$ & $\begin{array}{c}\text { Resistência } \\
\text { à compressão (MPa) }\end{array}$ \\
\hline 0,48 & 9,2 & 1,5 \\
\hline 0,50 & 9 & 1,4 \\
\hline 0,52 & 8,7 & 1,2 \\
\hline 0,54 & 8,4 & 1,2 \\
\hline
\end{tabular}




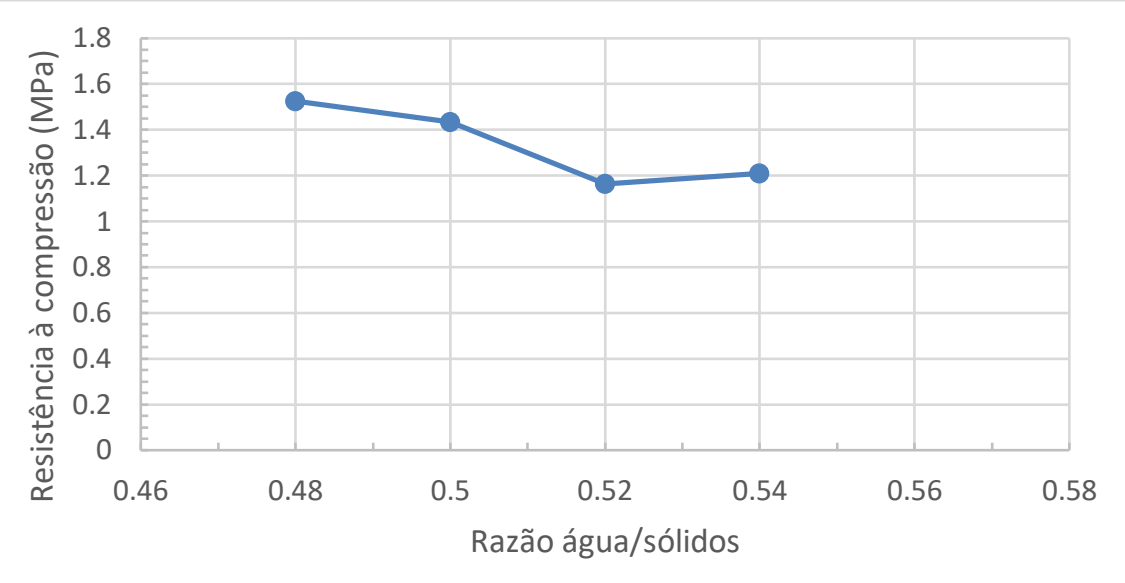

Figura 3. Resistência à compressão em função da razão água/sólidos para razão ativador/precursor igual a 0,5 .

A argamassa K0,5/0,56 foi descartada por não apresentar resistência considerável. Os resultados mostram a tendência de diminuição da resistência mecânica com o aumento da razão água/sólidos (diminuição da molaridade da solução ativadora).

De maneira geral, o aumento da resistência à compressão com o aumento da molaridade e com a diminuição da razão ativador/precursor estão coerentes com os encontrados por Somna et. al. [5] e Ravikumar et. al. [6], resguardadas as diferenças nos precursores e ativadores.

\section{CONCLUSÔES}

De acordo com os resultados obtidos, para as argamassas geopoliméricas estudadas e os materiais e métodos utilizados, é possível concluir:

- A resistência à compressão aumenta com o aumento da molaridade da solução ativadora;

- A resistência à compressão aumenta com a diminuição da razão ativador/precursor;

- A resistência à compressão é compatível com as necessárias para argamassas de múltiplo uso.

Vale a pena ressaltar que são necessários mais estudos das variáveis envolvidas para se obter uma composição ideal, com resistência à compressão mais elevada.

\section{REFERÊNCIAS}

1 Sindicato Nacional da Indústria do Cimento. Produção Regional 2016. 2017 [acesso em 12 de junho. 2017]. Disponível em http://www.snic.org.br.

2 Provis JL, Van Deventer JSJ. Geopolymers - Structure, processing, properties and industrial applications, Woodhead Publishing Limited. 2009.

3 De Weerdt K. Geopolymers - State of the art. COin Project report № 37. 2011.

4 Souza PSL. Verificação da influência do uso de metacaulim de alta reatividade nas propriedades mecânicas do concreto de alta resistência. Tese - Universidade Federal do Rio Grande do Sul. Porto Alegre. 2003.

5 Somna K, Jaturapitakkul C, Kajitvichyanukul P, Chindaprasirt P. $\mathrm{NaOH}$ - activated ground fly ash geopolymer cured at ambiente temperature. Fuel. 2011 [acesso em 23 de maio. 2017]. Disponível em http://www.elsevier.com/locate/fuel. 
6 Ravikumar D, Peethamparan S, Neithalath N. Structure and strength of $\mathrm{NaOH}$ activated concretes containing fly ash or GGBFS as the sole binder.Cement \& Concrete Composites. 2010; 32: 399-410. 